\title{
Giving drugs per rectum for systemic effect
}

Suppositories were used as early as $1500 \mathrm{BC}$ by the Egyptians for the local treatment of haemorrhoids and constipation. These suppositories were made by rolling conical chips of wood or bone in honey in which a drug had been boiled until the solution was nearly solid. A thousand years later Hippocrates described the use of a suppository made of anise, myrrh, goose grease, and honey as a specific relief for asthma, but it was in Britain in the seventeenth century that the word 'suppositorium' was first used. $^{1}$

The Egyptian technique of using inspissated honey was used until the end of the seventeenth century. Cocoa butter was then used as it liquefied readily on warming and could be mixed with various ingredients; it quickly formed a hard wax and yet conveniently dissolved in the rectum. It was in general use for the next two hundred years, until the introduction of water soluble polyethylene glycols. The importance of the base used in the formulation of a suppository has been reported over the past 20 years.

\section{Acceptability to the patient}

Suppositories are commonly used in certain parts of Europe, but not in the United Kingdom. It is clear from the amount that has been written on the subject that the cultural influences of the parent, the child, and the doctor all have an effect on the acceptability of the treatment. Although it is often difficult to get young children to take their medicine by mouth there is no evidence to suggest that compliance is improved if suppositories are prescribed.

\section{Indications for giving drugs through the rectum}

There are several circumstances under which it may be preferable to give a drug through the rectum as opposed to intramuscularly or intravenously; these include circumstances in which the patient is unconscious; nauseated or vomiting; or if oral intake is forbidden immediately before operation. First pass elimination in drugs with a high hepatic clearance may also be avoided in this way.

\section{Absorption from the rectum}

The rectum is drained by the superior, middle, and inferior rectal veins. The superior rectal vein drains through the inferior mesenteric vein into the portal vein, whereas the inferior and middle rectal veins drain directly into the inferior vena cava through the internal pudendal and internal iliac veins. There are extensive anastomoses between the rectal veins.

When a drug is taken orally and absorbed from the gastrointestinal tract, it enters the portal vein and is metabolised in the liver. If it is one which is principally broken down in the liver-for example, morphine, hydralazine, or propranolol, it does not achieve its optimal effect when taken orally. If it is given through the rectum however, better absorption and greater systemic effect may be achieved.

The mechanism of rectal absorption is probably similar to that of the upper part of the gastrointestinal tract, despite differences in $\mathrm{pH}$, surface area, and fluid content. The use of surfactants can increase the amount of absorption of a drug from the rectum; they can, however, damage the rectal mucosa and so their use requires further investigation. The effects of different pharmaceutical formulations of the suppository may also affect the availability of the drug, and this has been particularly well documented concerning paracetamol. ${ }^{2}$ A practical problem with the use of suppositories is premature expulsion, which may result in inadequate absorption.

\section{Specific drugs}

Anticonvulsants. A solution of diazepam given rectally is effective for treating convulsions in children, therapeutic plasma concentrations being obtained within four minutes. ${ }^{3}$ There is a considerable delay in the achievement of a therapeutic plasma concentration of diazepam after the use of suppositories, and with the introduction of rectal solutions there is no indication to use suppositories. The two major indications for the use of diazepam solution rectally in children are by parents at home for children who suffer from either febrile convulsions or recurrent, poorly controlled epilepsy, or by a doctor when immediate intravenous access is not technically possible. It is preferable to give diazepam intravenously in a paediatric unit or in an accident and emergency department, as therapeutic plasma concentrations are more readily achieved by this route. Although clonazepam can be administered rectally, it takes up to 20 minutes for 
therapeutic plasma concentrations to be achieved.

Paraldehyde used to be given rectally in the United States of America, but we now know that rectal absorption of paraldehyde is considerably slower than if it is given either intramuscularly or orally and it is therefore unsuitable for treating epilepsy. Paraldehyde can also irritate the rectum and large intestine when given rectally, and decomposed paraldehyde has caused perforation of the large bowel. It is far safer to administer it as a deep intramuscular injection.

Antibiotics. Metronidazole suppositories are effective in reducing the postoperative anaerobic wound infections in children with appendicitis. ${ }^{4}$ The suppositories are considerably cheaper than the intravenous infusion, and pharmacokinetic studies have shown that they produce therapeutic plasma concentrations. If, however, the child has an intravenous line, it is preferable for the drug to be given intravenously.

Paracetamol. Paracetamol is used mainly as an antipyretic in children. Although paracetamol suppositories are not commercially available, most hospital pharmacies produce their own. The formulation of the suppository influences the availability of the paracetamol, and there are few data on the biovailability of the paracetamol in suppositories in different hospitals. The major clinical indication for giving paracetamol rectally is if a child is admitted with an acute febrile convulsion, and the paracetamol can be administered before the child wakes up. Paracetamol is also useful in suppository form for children who have fevers associated with vomiting.
Other drugs. Theoretically, morphine is an ideal drug to be given rectally as it is extremely well absorbed. A pharmacokinetic study following rectal morphine $(0.15 \mathrm{mg} / \mathrm{kg})$ in propylene glycol, however, showed plasma concentrations that were inadequate for pain relief. ${ }^{5}$ Rectal theophylline has been used for children with asthma, but with the advent of oral slow release theophylline preparations suppositories have been superseded. There have been several reports of one or more drugs given rectally as premedication before operation. 56 Unfortunately, little data were given on the acceptability of the rectal compared with the intramuscular route.

\section{References}

1 Senior N. Review of rectal suppositories. 1. Formulation and manufacture. Pharmaceutical Journal 1969:203:703-6.

2 de Boer AG, Moolenaar F, de Leede LGJ, Breimer DD. Rectal drug administration: clinical pharmacokinetic considerations. Clin Pharmacokinet 1982;7:285-311

${ }^{3}$ Knudsen FU. Plasma diazepam in infants after rectal administration in solution and by suppository. Acta Paediatr Scand 1977;66:563-7.

${ }^{4}$ Ford WDA, MacKellar A, Richardson CJL. Pre- and postoperative rectal metronidazole for the prevention of wound infection in childhood appendicitis. J Pediatr Surg 1980;15: $160-3$

${ }^{5}$ Lindahl S, Olsson AK. Thomson D. Rectal premedication in children. Use of diazepam, morphine and hyoscine. Anaesthesia 1981:36:376-9.

' Lindsay WA, Shepherd J. Plasma levels of thiopentone after premedication with rectal suppositories in young children. $\mathrm{Br} \mathrm{J}$ Anaesth 1969:41:977-84.

I A Choonara

Department of Paediatrics and Child Health, St James's University Hospital, Leeds LS9 $7 T F$ 